\title{
The hot and cool component of the symbiotic nova SMC $3^{\star}$
}

\section{A supersoft X-ray variable and a small-amplitude red variable}

\begin{abstract}
P. Kahabka ${ }^{\star \star}$
Sternwarte Universität Bonn, Auf dem Hügel 71, 53121 Bonn, Germany

Received 9 December 2002 / Accepted 26 November 2003

Abstract. The $\sim 6$ year supersoft X-ray lightcurve of the symbiotic nova SMC 3 (=RX J0048.4-7332) in the Small Magellanic Cloud is derived from archival ROSAT PSPC and HRI data. It shows one deep X-ray eclipse during which the count rate decreased by a factor of $\gtrsim 80$. In $M A C H O B$-band data sinusoidal variation is found with a quasi-periodicity of $\sim 4$ years. The minimum of the $B$-band flux occurs during the X-ray eclipse. In $O G L E$ II I-band observations performed after the ROSAT observations we detect $110 \pm 2$ day oscillations which we interpret as pulsations of the M0 giant star in the symbiotic system. The observed duration of the supersoft X-ray eclipse of $\sim 0.4-1.8$ years is explained by the occultation of the white dwarf by the giant companion with an orbital period of $\sim(4.0-4.8)$ years and a strong wind blown from its surface with a mass loss rate of $\sim(2.6-8.2) \times 10^{-7} M_{\odot} \mathrm{yr}^{-1}$, assuming that $\sim(1-3.5) \%$ of the ionized phase is neutral (e.g. due to dust) and assuming a terminal velocity of $\sim 30 \mathrm{~km} \mathrm{~s}^{-1}$. The $\sim 4$ year quasi-periodicity found in the optical is explained as the binary orbital period of the system. It is less likely that it reflects the activity (or mass-loss) time scale of the red giant star. A $\sim(700-800)$ day quasi-periodicity found in the $O G L E I I$ and $M A C H O$ data is explained as the first harmonic of a binary orbital cycle. SMC 3 therefore may be classified as a small-amplitude red variable star (SARV). The hot star most likely is in a state of steady nuclear burning with an accretion rate somewhat below the upper critical value of $\sim 10^{-7} M_{\odot} \mathrm{yr}^{-1}$.
\end{abstract}

Key words. galaxies: Magellanic Clouds - stars: binaries: symbiotic - stars: individual: RX J0048.4-7332 (=SMC 3) stars: mass-loss - X-rays: stars

\section{Introduction}

RX J0048.4-7332 was discovered in the ROSAT all-sky-survey data (Kahabka \& Pietsch 1993). It has been optically identified with the symbiotic star SMC 3 in the Small Magellanic Cloud (Morgan 1992). The optical star is of spectral type M0. The hot component had a comparatively "mild" outburst sometime between December 1980 and November 1981, increasing by 3 mag in the $U$ band. No increase has been detected in the infrared. The intensity of the hot component has stayed nearly constant since this outburst (Morgan 1992). ROSAT PSPC observations have been performed from November 1991 to November 1993. The source has been classified as a supersoft X-ray source due to the softness of the X-ray spectrum (Kahabka et al. 1994). From the spectral modeling of the ROSAT PSPC observation performed in April 1992 by Jordan et al. (1996) the X-ray spectrum is consistent with a white dwarf with a mass of $\sim 0.8 M_{\odot}$ having steady nuclear burning on the surface and which has a strong Wolf-Rayet type wind with a mass-loss rate of $\sim 10^{-6} M_{\odot} \mathrm{yr}^{-1}$. An IUE observation of SMC 3 performed in April 1993 by Vogel \& Morgan (1994)

* Based on archival data from the ROSAT, OGLE-II and MACHO project.

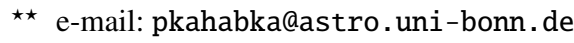

shows an enrichment of $\mathrm{N}$ similar to galactic novae, which was most likely related to a possible recent thermonuclear event connected with the optical outburst. A pronounced Si overabundance deduced from the IUE spectrum was explained by collisional ionization due to a high-density $\left(n_{\mathrm{e}} \geq 10^{9} \mathrm{~cm}^{-3}\right)$ symbiotic nebula in the system (Nussbaumer \& Stencel 1987). Such a nebula of size $\sim 1$ AU would result in a line of sight absorbing column in agreement with the H-column density of $\sim 10^{21} \mathrm{H}-$ atoms $\mathrm{cm}^{-2}$ deduced from the X-ray observations.

In Sects. 2 and 3 the ROSAT X-ray, OGLE II I-band and MACHO $B$-band lightcurve is derived. In Sect. 4 a dip found in the X-ray lightcurve is explained as an eclipse of the X-ray source by the red giant companion star. From the modeling of the orbital lightcurve in X-rays, constraints are derived for the orbital period and the the mass-loss rate of the giant star.

\section{The hot component}

RX J0048.4-7332 is a supersoft X-ray source (cf. Kahabka \& van den Heuvel 1997; Kahabka \& van den Heuvel 2003 for a review of supersoft sources). The X-ray source coincides in position with the symbiotic nova SMC 3 (Morgan 1992). From the HRI observation an X-ray position $\operatorname{RA}(2000)=0^{\mathrm{h}} 48^{\mathrm{m}} 19.3^{\mathrm{s}}, \operatorname{Dec}(2000)=-73^{\circ} 31^{\prime} 53^{\prime \prime}$ is derived. 
The X-ray position agrees within $\sim 10^{\prime \prime}$ with the optical position $\mathrm{RA}(2000)=0^{\mathrm{h}} 48^{\mathrm{m}} 19.9^{\mathrm{s}}, \operatorname{Dec}(2000)=-73^{\circ} 31^{\prime} 54.9^{\prime \prime}$ (Munari \& Zwitter 2002). The ROSAT name of the source, RX J0048.4-7332 is based on less accurate positional information (cf. Kahabka et al. 1994) than has been derived with the HRI. RX J0048.4-7332 has not been detected with the Einstein satellite but may have been missed due to its location in a poorly exposed area. As the Einstein observations in the direction of the Small Magellanic Cloud were performed from April 1979 to April 1980, i.e. before the optical outburst which happened between December 1980 and November 1981, this would be consistent with the fact that SMC 3 was not a strong supersoft X-ray source during the Einstein observation. This is in agreement with a symbiotic nova outburst.

\subsection{X-ray lightcurve}

There have been 8 ROSAT PSPC observations (including one all-sky survey observation in October 1990) and 8 ROSAT HRI observations performed in the direction of the symbiotic nova. The PSPC observations were performed from 21 October 1990 to 30 November 1993 and the HRI observations from 19 April 1994 to 19 November 1996. The observational data were retrieved from the public ROSAT archive. In Table 1 the X-ray observations performed in the direction of SMC 3 are given together with the ROSAT PSPC and HRI count rate.

The count rate has been determined for each observation making use of the EXSAS data analysis programs (Zimmermann et al. 1994). For each observation an image and an exposure map were created which (for the PSPC) takes also source obscuration due to the detector struts into account. The $H R I$ count rate was multiplied by a factor of 3 to account for the difference in the effective area of the PSPC and the HRI. The X-ray lightcurve is given in Fig. 1.

It shows a pronounced dip during which the source count rate decreased by a factor of $\gtrsim 80$. After the dip the source count rate returned to a level comparable to before the dip although the absolute intensity level may be somewhat uncertain. It is not clear whether the source enters only temporarily into an X-ray dip or whether X-ray dips also happen before and after this observation. An argument for the second option may be that during an observation in April 1998 (cf. Table 1) the source was not detected (however, it was at the very outer edge of the detector). It may have entered into another dip.

\subsection{X-ray spectrum}

The X-ray spectrum is supersoft but apparently deviates from a blackbody type spectrum. Sophisticated non-LTE modeling of the spectral distribution has been performed by Jordan et al. (1996) on the April 1992 X-ray observation of SMC 3. In addition they made use of the UV observation performed with IUE on 30 April 1993, which is one year after the ROSAT observation. They derived with their model a temperature for the hot component of $>2.6 \times 10^{5} \mathrm{~K}$ and a luminosity of $\sim 10^{4} L_{\odot}$. The white dwarf mass estimated was $\sim 0.8 M_{\odot}$. Although Jordan et al. (1996) used a wide parameter set to explore a common
Table 1. ROSAT PSPC and HRI observations in the direction of SMC 3. The ROSAT observation ID (with p designating the PSPC and $\mathrm{h}$ the $H R I$ ), the observation time interval, the exposure of the observation, the off-axis angle of the source in the detector, and the measured count rate is given. For the HRI observations the count rate has been multiplied by a factor of three to account for the difference in effective area between the PSPC and the HRI.

\begin{tabular}{|c|c|c|c|}
\hline ID & \multicolumn{2}{|c|}{$\begin{array}{l}\text { Time start } \\
\text { (UT) }\end{array}$} & $\begin{array}{l}\text { Time end } \\
\text { (UT) }\end{array}$ \\
\hline$\overline{\operatorname{RASS}^{a}}$ & \multicolumn{2}{|c|}{$21-10-90-17: 24$} & $26-10-90-11: 06$ \\
\hline $600196 p-0$ & \multicolumn{2}{|c|}{ 09-10-91-03:07 } & $09-10-91-04: 48$ \\
\hline $600196 \mathrm{p}-1$ & \multicolumn{2}{|c|}{$25-04-92-11: 52$} & 22-04-92-21:36 \\
\hline $600454 p$ & \multicolumn{2}{|c|}{ 05-12-92-23:59 } & $08-12-92-01: 40$ \\
\hline $600454 p-1$ & \multicolumn{2}{|c|}{ 05-04-93-06:24 } & $26-04-93-23: 31$ \\
\hline $600453 p$ & \multicolumn{2}{|c|}{ 09-05-93-07:33 } & $12-05-93-20: 13$ \\
\hline $500249 p$ & \multicolumn{2}{|c|}{$05-11-93-23: 31$} & 09-11-93-02:37 \\
\hline $500251 p$ & \multicolumn{2}{|c|}{$30-11-93-05: 41$} & $30-11-93-07: 35$ \\
\hline $400340 \mathrm{~h}$ & \multicolumn{2}{|c|}{ 19-04-94-19:49 } & 21-04-94-10:17 \\
\hline $400335 h$ & \multicolumn{2}{|c|}{$19-04-94-11: 35$} & $08-05-94-04: 28$ \\
\hline $400340 \mathrm{~h}-1$ & \multicolumn{2}{|c|}{$13-10-94-17: 24$} & 21-10-94-06:34 \\
\hline $500419 \mathrm{~h}$ & \multicolumn{2}{|c|}{$18-05-95-10: 23$} & $05-06-95-23: 55$ \\
\hline $500419 \mathrm{~h}-1$ & \multicolumn{2}{|c|}{$03-12-95-10: 23$} & $03-12-95-11: 32$ \\
\hline $600812 \mathrm{~h}$ & \multicolumn{2}{|c|}{$26-04-96-18: 04$} & $07-06-96-13: 30$ \\
\hline $300513 \mathrm{~h}$ & \multicolumn{2}{|c|}{$19-10-96-11: 08$} & $19-10-96-11: 40$ \\
\hline $600812 \mathrm{~h}-1$ & \multicolumn{2}{|c|}{$11-11-96-05: 01$} & $14-11-96-15: 39$ \\
\hline $601045 \mathrm{~h}$ & \multicolumn{2}{|c|}{ 05-04-98-22:41 } & 23-04-98-04:22 \\
\hline ID & $\begin{array}{l}\text { xposure } \\
(\mathrm{ks})\end{array}$ & $\begin{array}{l}\text { off-axis } \\
\text { (arcmin) }\end{array}$ & $\begin{array}{c}\text { count rate } \\
\left(\mathrm{s}^{-1}\right)\end{array}$ \\
\hline $\mathrm{SS}^{a}$ & 11:06 & 0.8 & $0.21 \pm 0.04$ \\
\hline $0196 p-0$ & 1.3 & 21 & $0.218 \pm 0.0161$ \\
\hline $0196 \mathrm{p}-1$ & 22.2 & 21 & $0.264 \pm 0.00429$ \\
\hline $0454 p$ & 9.7 & 24 & $0.230 \pm 0.00610$ \\
\hline $0454 p-1$ & 8.3 & 24 & $0.171 \pm 0.00562$ \\
\hline $0453 p$ & 17.6 & 53 & $0.0827 \pm 0.00516$ \\
\hline $0249 p$ & 19.2 & 21 & $0.00397 \pm 0.00138$ \\
\hline $0251 \mathrm{p}$ & 2.1 & 15 & $0.00731 \pm 0.00278$ \\
\hline $0340 \mathrm{~h}$ & 2.6 & 15 & $<0.00564^{b}$ \\
\hline $0335 \mathrm{~h}$ & 17.6 & & $<0.0106^{b}$ \\
\hline 0340h-1 & 2.6 & 15 & $0.0854 \pm 0.0185$ \\
\hline $0419 \mathrm{~h}$ & 11.3 & 15 & $0.158 \pm 0.0116$ \\
\hline 0419h-1 & 4.3 & 15 & $0.202 \pm 0.0217$ \\
\hline $0812 \mathrm{~h}$ & 11.5 & 4 & $0.217 \pm 0.0216$ \\
\hline $00513 \mathrm{~h}$ & 1.6 & 0 & $0.247 \pm 0.0313$ \\
\hline 00812h-1 & 17.1 & 4 & $0.202 \pm 0.00858$ \\
\hline $01045 \mathrm{~h}$ & 9.5 & 20 & $<0.019^{\mathrm{c}}$ \\
\hline
\end{tabular}

Remarks: ${ }^{a}$ from Kahabka et al. (1994), ${ }^{b} 4 \sigma$ upper limit, ${ }^{c} 3 \sigma$ upper limit derived in a $78^{\prime \prime}$ circle at an off-axis angle of 18.7 .

solution for the fit of the ROSAT and IUE data they did not arrive at a perfect fit. The temperature derived from the ROSAT observations of $>2.6 \times 10^{5} \mathrm{~K}$ appeared to be too high to account for the UV flux. From the He II recombination analysis a blackbody temperature of $10^{5} \mathrm{~K}$ and a luminosity of $2 \times 10^{37} \mathrm{erg} \mathrm{s}^{-1}$ were derived by Jordan et al. (1996). From Fig. 1 a solution for this discrepancy may be clear: the supersoft X-ray source was during the ROSAT observation at the flux peak in the lightcurve and during the $I U E$ observation had entered into a deep X-ray dip. Thus a decrease in the observed flux occurred between 

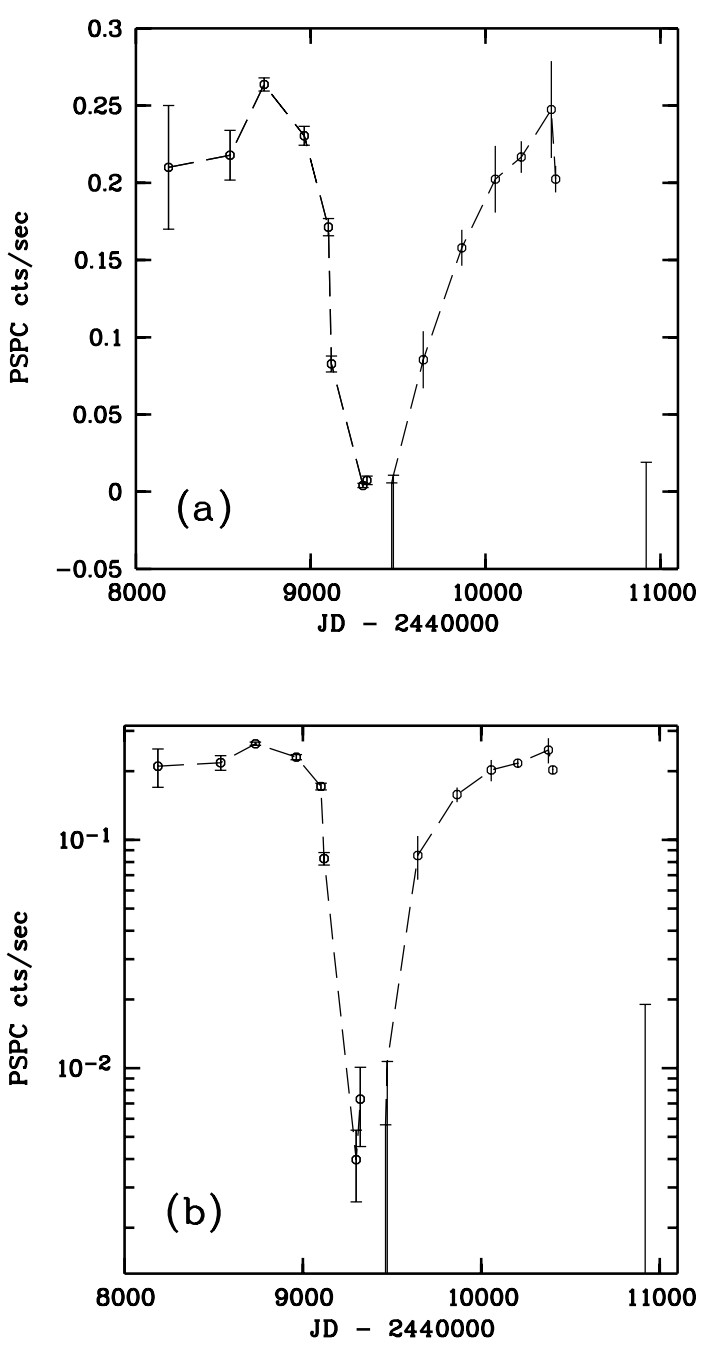

Fig. 1. ROSAT PSPC and HRI lightcurve of RX J0048.4-7332. A linear scale a) and a logarithmic scale b) is chosen for the count rate (cf. Table 1).

both observations and a fit common to the ROSAT and IUE data is problematic. In Fig. 2 the value for the hardness ratio derived during the observations is shown.

During the bright part of the lightcurve the hardness ratio $H R=(S 2-S 1) /(S 2+S 1)$ with $S 1$ and $S 2$ the counts in the (8-41) and (41-90) ROSAT PSPC band respectively is in the range -0.85 to -0.875 which would be consistent with a blackbody temperature of $\sim 30-35 \mathrm{eV}$ (assuming there is no $\mathrm{C} \mathrm{V}$ edge). Using a wider range for the hardness ratio of -0.8 to -0.9 and allowing for a $\mathrm{C} \mathrm{V}$ edge with $\tau \lesssim 2$ then the blackbody temperature would be $25-45 \mathrm{eV}$. There is the trend of a slight spectral hardening which can be explained by an increase of the absorbing column density from $\sim 3$ to $\sim 8 \times 10^{20} \mathrm{~cm}^{-2}$. This is in agreement with the dependence of the absorbing column density with orbital phase as deduced from the fitting of the eclipse lightcurve (cf. Fig. 7). During mid-eclipse the formal errors of the hardness ratios are too large to derive reliable values for the temperature and absorbing column density.
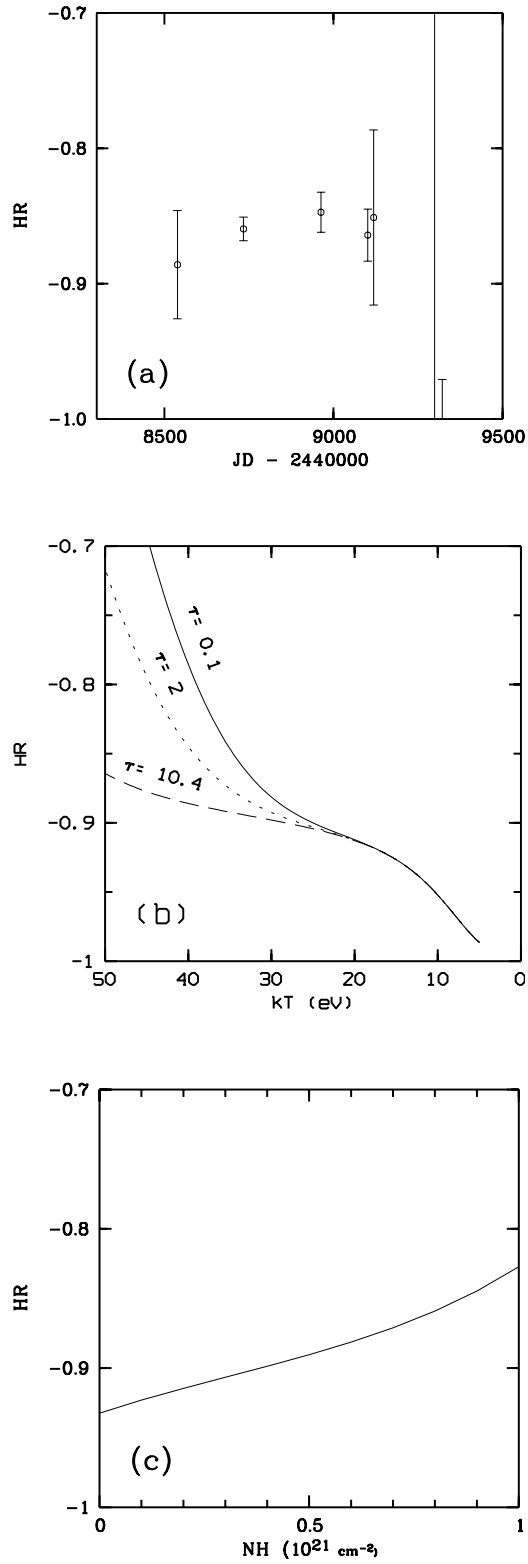

Fig. 2. a) Hardness ratio $H R=(S 2-S 1) /(S 2+S 1)$, with $S 1$ and $S 2$ the counts in the 8-41 and 41-90 ROSAT PSPC band respectively as a function of time during the ROSAT PSPC observations of SMC 3 (cf. Fig. 1 and Table 1). b) Same hardness ratio derived from simulated spectra as a function of the blackbody temperature including a $\mathrm{CV}$ edge with an optical depth of $0.1,2$, and 10.4 and assuming columns of 3 and $6 \times 10^{20} \mathrm{~cm}^{-2}$ with galactic and reduced metallicities $(-0.6$ dex $)$. c) Same hardness ratio derived from simulated spectra with a blackbody temperature of $30 \mathrm{eV}$ (and no $\mathrm{C} \mathrm{V}$ edge) as a function of the absorbing column density.

\section{The cool component}

The cool component of the symbiotic nova SMC 3 has been classified an s-type (stellar-type) symbiotic (Mürset et al. 1994) which is determined in the infrared by the photospheric emission of a normal red giant star. SMC 3 is brighter than a normal giant of luminosity class III (cf. discussion in Morgan 1992, see also Dumm \& Schild 1998); this means it has a radius which is larger than for such a giant. 


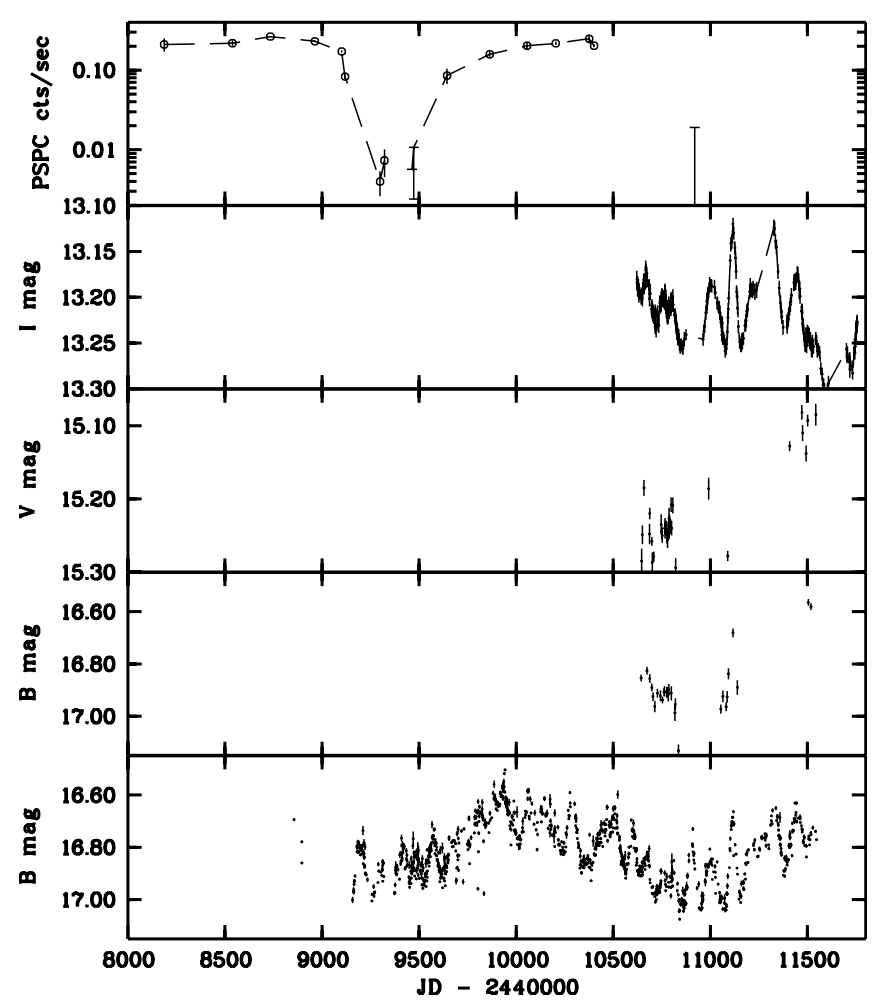

Fig. 3. ROSAT lightcurve (upper panel), $O G L E I I$ calibrated $I, V$, and $B$-band (second to fourth panel), and $M A C H O B$-band lightcurve (uncalibrated lightcurve, adjusted to the $O G L E$ II $B$-band light curve) of SMC 3.

\subsection{OGLE lightcurve}

SMC 3 is located in the OGLE II field SMC_SC4 (Udalski et al. 1998; Udalski et al. 1997). The OGLE counterpart has the designation OGLE 004820.06-733151.7. The accuracy of the OGLE position is $\sim 0.7^{\prime \prime}$ (Udalski, private communication). The OGLE source is contained in the catalog of SMC variable stars found with $O G L E$ II. In Fig. 3 OGLE II (I, V, and $B$-band) observations of SMC 3 performed after the ROSAT observations from 1997 for $\sim 3$ years are shown.

A FFT analysis has been applied to the OGLE II I-band data (Lomb 1976; Scargle 1982). Oscillations with a period of $110 \pm 2$ days are present (cf. Fig. 4). Such oscillations can be explained as pulsations of the red giant star. Such smallamplitude red variables (SARVs) generally of spectral type M3III to M6III, are pulsating, apparently irregularly with time scales of 50-200 days and with amplitudes of 0.1 to a magnitude or more. SARVs also show long periods (hundreds or thousands of days) whose nature is unclear. There is a second peak in the periodogram of SMC 3 with a quasi-periodicity of 600-750 days which may be such a long cycle although the coverage of our data is too short to establish the cycle. The SARVs are closely related to the Miras which are on the AGB and which have longer pulsational periods and larger amplitudes (Percy et al. 1996).

\subsection{MACHO lightcurve}

The MACHO B-band lightcurve has been retrieved with the interactive lightcurve browser ${ }^{1}$. The uncalibrated lightcurve was used, shifted in magnitude to match in the region of overlap with the $O G L E$ II $B$-band lightcurve. A FFT analysis was applied to the MACHO $B$-band data. The lightcurve is quasisinusoidal with a quasi-periodicity of $\sim 4$ years (cf. Figs. 3 and 4). Two minima of the sinusoidal modulation are found, one minimum coincides with the X-ray eclipse, the second minimum occurs during the supposed second X-ray eclipse, which would argue for the occurance of a second eclipse. In addition a shorter period of $\sim 106 \pm 2$ days is found in the lightcurve (especially for times JD $>2450000$ ) which matches the period of $\sim 110 \pm 2$ days found in the OGLE II lightcurve. The lightcurve is clearly multi-periodic and SMC 3 can be classified as a semiregular variable (cf. Lebzelter et al. 2002)

\section{Variability}

The variability observed in SMC 3 during the $\sim 6$ year observational ROSAT campaign with a typical time spacing of $\sim 0.5$ years is the first detection of X-ray variability in a supersoft X-ray source with such a long observational record. The time scale involved is $\sim 1000$ days and much longer than the variability observed in the supersoft LMC transient RX J0513.9-6951 of $\sim 30$ days (Reinsch et al. 2000). A different scenario is considered for SMC 3 to account for the $\mathrm{X}$-ray variability; an eclipse of the white dwarf by the giant star and its wind. In the case of the symbiotic nova SMC 3 the mass-loss is due to stellar wind. A (large) accretion disk is not expected to be present in this system, in comparison to RX J0513.9-6951 where the mass-transfer occurs via an accretion disk. Mass-loss variations of the donor star in symbiotic systems may be due to (radial) pulsations of the red giant (or the AGB) star with typical periods of a few hundred days. In addition long cycles (of several hundreds or thousands of days) have been observed. Such long massloss cycles may drive the white dwarf envelope into quasioscillations as long as the cycle length is comparable to or longer than the Kelvin-Helmholtz time of the white dwarf envelope $\tau_{\mathrm{KH}} \approx 3100 \frac{M_{\mathrm{WD}}}{\left(M_{\odot}\right)} \frac{M_{\mathrm{env}}}{\left(10^{-5} M_{\odot}\right)}\left(\frac{R_{\mathrm{WD}}}{\left(10^{9} \mathrm{~cm}\right)}\right)^{-1}\left(\frac{L_{\mathrm{WD}}}{\left(10^{37} \mathrm{erg} \mathrm{s}^{-1}\right)}\right)^{-1}$ days, which is $\tau_{\mathrm{KH}} \sim 1000$ days for a $0.8 M_{\odot}$ white dwarf with an envelope mass of $M_{\text {env }} \sim(1-2) \times 10^{-5} M_{\odot}$ (cf. Kahabka 1996; Kahabka \& van den Heuvel 2003) and roughly consistent with the observed duration of the dip. But here a different scenario will be considered to explain the X-ray dip, the eclipse of the white dwarf by the red giant star and its wind. SMC 3, namely entered into an X-ray off-state (eclipse) for $\sim 200$ days (from November 1993 to April 1994). It then recovered from the eclipse and returned to its nominal flux level. The width of the dip is $\sim 600$ days. The eclipse ingress and egress is gradual and a strong wind lost from the surface of the giant star is required to explain the shape of the eclipse lightcurve (cf. Sect. 4.3 for a modeling of the X-ray eclipse lightcurve).

\footnotetext{
${ }^{1}$ http://www.macho.mcmaster.ca/Data/MachoData.html
} 

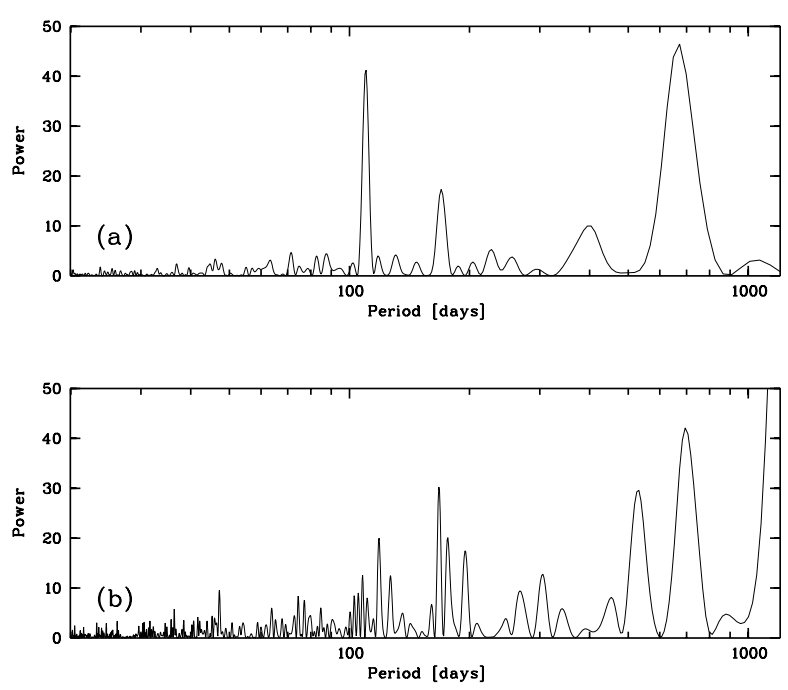

Fig. 4. a) Power spectrum of the calibrated $I$-band $O G L E I I$ lightcurve of SMC 3. Peaks with high power are found for periods of $\sim 110$ and $\sim 670$ days. b) Power spectrum of the MACHO B-band lightcurve of SMC 3. Peaks with high power are found at periods of of $\sim 120$ days and $\sim 670$ days.

Table 2. Periods (and power) derived from the FFT analysis of the OGLE II and Macho lightcurves of SMC 3.

\begin{tabular}{cccc}
\hline \hline \multicolumn{2}{c}{ Ogle II } & \multicolumn{2}{c}{ Macho } \\
Period & Power & Period & Power \\
\hline $110 \pm_{3}^{2}$ & 41 & $118 \pm_{1}^{2}$ & 20 \\
$170 \pm_{5}^{5}$ & 17 & $168 \pm_{2}^{2}$ & 30 \\
& & $528 \pm \pm_{28}^{30}$ & 28 \\
$670 \pm{ }_{65}^{77}$ & 46 & $670 \pm \pm_{20}^{80}$ & 42 \\
& & $1300 \pm_{150}^{150^{a}}$ & 42 \\
\hline
\end{tabular}

${ }^{a} \sim 1 / 2$ the period covered by the observation. The optical minima are separated by $\sim 1500$ days.

\subsection{Timescales and periodicities}

High mass-loss rates $\left(\dot{M} \gtrsim 10^{-8} M_{\odot} \mathrm{yr}^{-1}\right)$ are expected from pulsating red giant stars (Kafatos et al. 1977). The 110 day (most likely pulsational) period in the OGLE II infrared data of SMC 3 points to such a high mass-loss rate of the red giant star. Such variables are known as small-amplitude red variables which also show long periods (of several hundreds to thousands of days), whose nature is unclear ${ }^{2}$.

There are two long periodicities of $\sim(700-800)$ days and $\sim 1500$ days found in the OGLE II and MACHO data which could be related to a long period in the red variable of SMC 3. For this $\sim(700-800)$ day variability and the $\sim 1500$ day variability found in the $M A C H O B$-band data a likely explanation in terms of a binary orbital modulation (eclipse) is given in Sect. 4.3. An alternative explanation would be e.g. magnetic activity of the red giant star (cf. Soker 2002). But the length of a

\footnotetext{
${ }^{2}$ It has been suggested that such a long period may be due to the binary orbit or due to a convectively induced oscillatory thermal mode (Percy et al. 2002).
}

magnetic cycle in red giant stars is much longer ( $>200$ years, Soker 2000). Still magnetic activity as the origin of this variability cannot be excluded as stochastic mass-loss may occur on time scales much shorter than a magnetic cycle. Icke et al. (1992) presented a scenario of "weak chaos" to account for the variability observed in long-period variables. Pulsations originating in the stellar interior propagate through the outer mantle of the star which acts as a driven oscillator giving rise to mass-loss from the star. With decreasing mass of the stellar mantle the response to stellar pulsations will become ever more chaotic. We note that the MACHO B-band lightcurve of SMC 3 quite closely matches the multi-periodic lightcurves of Icke et al. (1992, see his Fig. 13), assuming weak chaos. The pulsations of the donor star of the symbiotic nova SMC 3 are still quite harmonic, as the periodogram derived over the OGLE II observations which cover a period of $\sim 3$ years shows a narrow peak at a period of $110 \pm 2$ days. The same result is achieved from a periodogram of the $M A C H O B$-band data over the last $\sim 4$ years of observation. This may mean that the 110 day pulsations are quite stable (harmonic) and the degree of chaos in this system is quite weak. This is consistent with the fact that the donor star of SMC 3 is still on the red giant branch and has not yet entered the asymptotic giant branch where the mantle of the star has decreased and the system enters into a more chaotic behavior with enhanced mass-loss.

\subsection{Binarity}

If the quasi-periodicity of the $B$-band flux of $\sim 4$ years would be connected to mass-loss variations of the red giant star and thus to the activity of the star, then the origin of such a long term activity would be unknown. It also is unknown whether there occur variations in the infrared, especially during the X-ray dip, which could indicate activity of the red giant star. A more likely explanation is binarity. An orbital period of $\sim 4$ years ( $\sim 1500$ days) is quite long for a symbiotic nova (cf. Mikolajewska 2003), as the red giant star will fill only a fraction $(\sim 30 \%)$ of its Roche lobe. The mass-transfer rate to the white dwarf would be mainly due to a stellar wind. An eclipse of the X-ray source is still possible as the absorbing column densities in the red giant wind are considerable $\left(\gtrsim 10^{21} \mathrm{~cm}^{-2}\right)$. The steadily nuclear-burning white dwarf could also be in an eccentric orbit around the red giant star (cf. Dumm et al. 1998 for BX Mon). Due to the orbital eccentricity, the white dwarf enters into a denser stellar wind during periastron which results in a larger absorbing column density, but such a scenario seems not to be required to explain the observed hardness ratios. The clock could also reside in the nuclear burning white dwarf, but a recurrence period of $\sim 4$ years would be too short (cf. Prialnik $\&$ Kovetz 1995). It is unlikely that the temperature variations inferred from the observed modulation in the $B$-band flux are due to X-ray irradiation (heating) of the donor star. Assuming an orbital period of $\sim 4$ years, the increase in temperature due to irradiation would be too low $(\sim 1.5 \%)$ to account for the temperature variation of $\sim 4.5 \%$ required to account for the observations. Assuming the 700-800 day quasi-period as the orbital period, then irradiation of the donor star by the hot white dwarf 


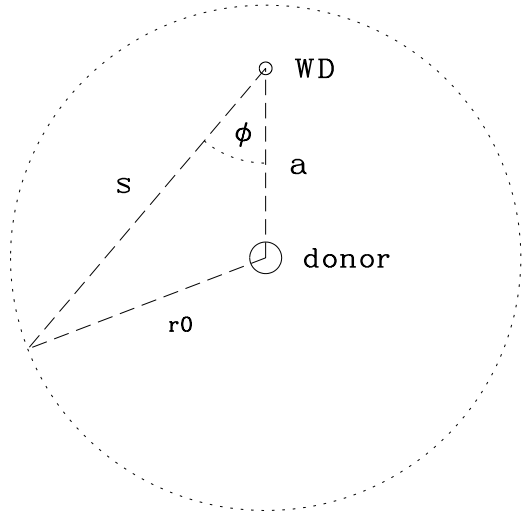

Fig. 5. Length of path $s$ of absorbing column towards the white dwarf and through the stellar wind of the donor. The phase angle $\phi$ is shown. The radius of the wind zone is $r_{0}$.

would lead to a temperature increase by $4.5 \%$ consistent with the observed value. But such a temperature variation should be observed as a change in spectral type which is not seen in the optical spectrum taken by Munari \& Zwitter (2002) during the X-ray minimum and by Morgan (1992) during a supposed optical maximum preceding the observed cycle.

The $~ 700-800$ day period most likely is the first harmonic of the $\sim 1500$ day quasi-period and could be due to ellipsoidal (tidal) distortion of the donor or due to stellar rotation (cf. Smith et al. 1997). Such an ellipsoidal distortion strongly depends on the fraction of the Roche lobe which is filled. The $B$ and $I$ lightcurves of SMC3 would qualitatively match the corresponding lightcurves of YY Herculis (Mikolajewska et al. 2002). But for the $\sim 1500$ day orbital period the giant would fill only a fraction of its Roche lobe and tidal distortion of the giant would not be very efficient.

\subsection{The $X$-ray eclipse}

Here the X-ray eclipse is modelled by the occultation of the nuclear-burning white dwarf by the companion star and by absorption due to a stellar wind from the companion star. We note that Dumm et al. (1999) use a somewhat different approach to determine the hydrogen absorbing column density for the symbiotic system SY Mus.

We assume a simple wind model making use of the radiatively driven wind model of Castor et al. (1975). The density then scales as

$n(r)=\frac{\dot{M}}{4 \pi m_{\mathrm{p}} v_{\infty}} \frac{1}{r^{2}\left(1-\frac{R}{r}\right)^{\beta}}$

with the proton mass $m_{\mathrm{p}}$, the terminal velocity $v_{\infty}$, the distance from the donor star $r$ and the radius of the donor star $R$. The length of the path $s$ from the white dwarf to the cut radius of the wind zone (assuming an inclination of $90^{\circ}$ ) can be expressed as (cf. Fig. 5)

$s=a \cos (\phi)+\sqrt{(a \cos (\phi))^{2}-\left(a^{2}-r_{0}^{2}\right)}$,

with the binary separation $a$, the radius of the wind zone $r_{0}$ and the phase angle $\phi$, which is related to the binary orbital phase $\varphi$ by $\varphi=\phi+\frac{a_{1}}{s} \sin \phi$, with $a_{1} M_{\mathrm{WD}}=a_{2} M_{\mathrm{donor}} \cdot \varphi \approx \phi$ for $s \gg a_{1}$. The system inclination $i$ can be taken into account by replacing $\phi$ with $\arccos (\cos (\phi) \sin (i))$. The absorbing gas column density of neutral hydrogen is determined from

$N_{\mathrm{H}}=\int_{s=0}^{s\left(r_{0}\right)} n(s) \mathrm{d} s$.

$n(s)$ can be expressed as

$n(s)=\frac{\dot{M}}{4 \pi m_{\mathrm{p}} v_{\infty}} \frac{1}{\left(u^{\frac{2}{\beta}}-R u^{\frac{2-\beta}{\beta}}\right)^{\beta}}$

with

$u^{2}=a^{2}+s^{2}-2 a s \cos (\phi)$.

We set $\beta=1$ (as is valid for mass-loss from cool stars, cf. Vogel 1991). From Eqs. (1) and (4) it follows that the absorbing gas column and the eclipse lightcurve depend on the ratio $\frac{\dot{M}}{v_{\infty}}$. This means that if the terminal velocity of the wind from the donor star (red giant) has been determined by other means (e.g. from the P Cygni profile of wind lines), then the mass-loss rate $\dot{M}$ of the donor star can be constrained. We use a value for the terminal velocity of the red giant star of $3 \times 10^{6} \mathrm{~cm} \mathrm{~s}^{-1}$ to constrain the mass-loss rate of the giant.

The absorbed PSPC count rate has been determined from simulations using a blackbody spectrum with $k T=30 \mathrm{eV}$, SMC reduced metallicities ( $-0.6 \mathrm{dex})$, a foreground galactic column of $3 \times 10^{20} \mathrm{~cm}^{-2}$ and an inclination of $60^{\circ}$ to $90^{\circ}$. For each data point of the observed lightcurve the column density of neutral and ionized hydrogen has been calculated using a threshold for the ionization parameter $\xi=\frac{L}{n s^{2}}$ of 1.0, with the luminosity $L$ (Kallman \& McCray 1982, see also Seaquist et al. 1984 for an approach to calculate the ionization front in a symbiotic X-ray binary). In the ionized gas phase an additional neutral (e.g. dust) phase has been assumed which would correspond to a dust to gas ratio of $0.01-0.035$ similar to dust to gas ratios found in the envelopes of M-type giants and Miras (Groenewegen et al. 1998; Knapp 1985). But we note that SMC 3 is an s-type symbiotic system of spectral type M0 which may not have a large amount of dust. Depending on the assumed value of the dust to gas ratio mass-loss rates in the range (2.6-8.2) $\times 10^{-7} M_{\odot} \mathrm{yr}^{-1}$ have been inferred assuming a terminal velocity of $3 \times 10^{6} \mathrm{~cm} \mathrm{~s}^{-1}$. The simulated orbital lightcurve has been compared in a chi-squared fit with the observed orbital lightcurve of SMC 3 (cf. Fig. 6 for the best-fit orbital lightcurve and Fig. 7 for the orbital dependence of the neutral hydrogen column density). In general the count rate error has been increased in the chi-squared fit to $0.15 \mathrm{~s}^{-1}$ for PSPC count rates $>0.3 \mathrm{~s}^{-1}$.

We determined an orbital period of $P_{\text {orb }}=4.4 \pm 0.4$ years and a mid-eclipse ephemeris (90\% confidence) of

$\mathrm{JD}_{\text {Min }}=2449360+(1600 \pm 140) \times E$.

The inclination of the system has to be high, $i \gtrsim 60^{\circ}$, in order to reproduce the deep dip in the orbital lightcurve and the absorbing gas columns inferred from the hardness ratios. The mean mass-loss rate inferred from this analysis is consistent with mass-loss rates derived for other giants in symbiotic systems (e.g. $\dot{M} \approx 5 \times 10^{-7} M_{\odot} \mathrm{yr}^{-1}$ for $\mathrm{SY}$ Mus, 


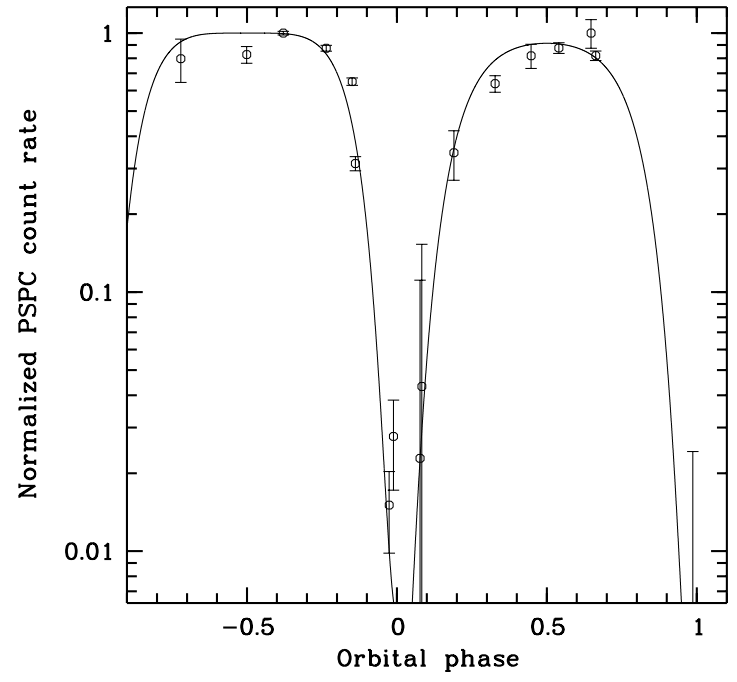

Fig. 6. Best-fit orbital lightcurve assuming occultation by the red giant donor star and absorption due to wind driven from its surface, assuming an inclination of $70^{\circ}$. The best-fit parameters are: Mass-loss rate $\dot{M}=1.6 \times 10^{19} \mathrm{~g} \mathrm{~s}^{-1}$, orbital period $P_{\text {orb }}=1600$ days, mid-eclipse time $T_{\text {ecl }}=$ JD 2449360 , binary separation $a=5.40 \times 10^{13} \mathrm{~cm}$, radius of wind zone $r_{0}=1.47 \times 10^{15} \mathrm{~cm}$ (kept fixed), lightcurve asymmetry parameter $\epsilon=0.825, M_{\mathrm{WD}}=0.8 M_{\odot}, M_{\mathrm{donor}}=3.0 M_{\odot}$ (kept fixed), ratio stellar radius to Roche lobe radius $R_{\mathrm{s}} / R_{\mathrm{L}}=0.3$ (kept fixed), $\chi / 13$ d.o.f. $=0.852$.

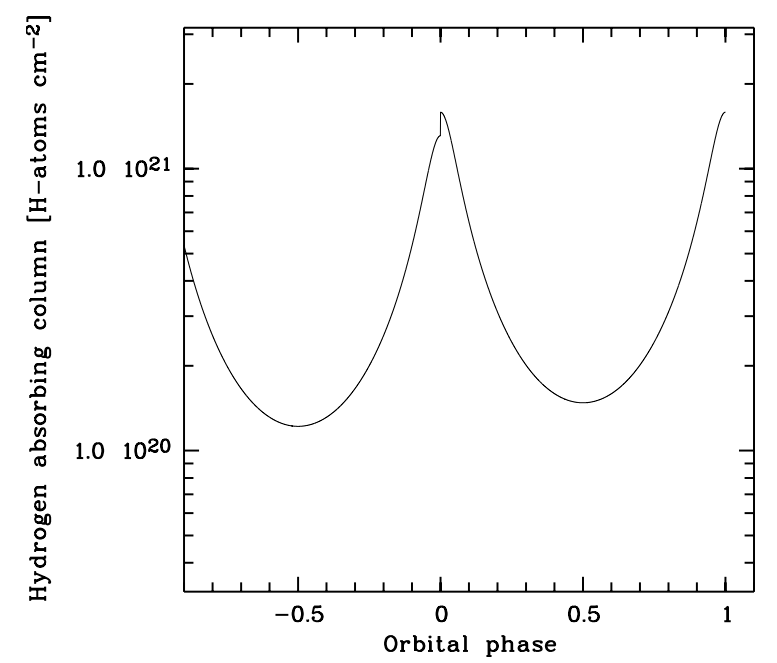

Fig. 7. Dependence of neutral hydrogen absorbing column density due to the red giant wind on the orbital phase, derived from the bestfit model lightcurve (cf. Fig. 6). An additional neutral (e.g. dust) phase with a fraction of 0.035 is assumed in the ionized component.

Dumm et al. 1999) and it is consistent with mass-loss rates inferred for symbiotic stars from radio observations (Seaquist et al. 1984). It is also consistent with the mass-loss rate pulsation period relation of M-type giants assuming a pulsational period of 110 days for SMC 3 (Kafatos et al. 1977). Such a high wind mass-loss rate should result in a high effective accretion rate onto the white dwarf. Assuming an efficiency of $\sim 7 \%$ (Sion et al. 2002) such an accretion rate would be $\sim(2-6) \times 10^{-8} M_{\odot} \mathrm{yr}^{-1}$ and somewhat below the steady nuclear burning rate of $6.5 \times 10^{-8} M_{\odot} \mathrm{yr}^{-1}$ for a $0.8 M_{\odot}$ white dwarf.

\section{Evolutionary considerations}

The reported outburst amplitude of SMC 3 was comparatively small ( 3 mag in the $U$ band). This may indicate a mild flash. According to Paczyński \& Rudak (1980) the flash strength decreases with increasing accretion rate. This could mean that the accretion rate is comparatively large in the symbiotic nova SMC 3. For accretion rates $\dot{M}_{\text {acc }}>\dot{M}_{\text {crit }}$, with the critical accretion rate $\dot{M}_{\text {crit }}=2.8 \times 10^{-7}+5.9 \times 10^{-7}\left(M_{\mathrm{c}} / M_{\odot}-1.0\right)$, the radius of the white dwarf envelope expands after the initial shell flash and no supersoft X-ray emission will be observed. For accretion rates $\dot{M}_{\text {crit }}>\dot{M}_{\text {acc }}>\dot{M}_{\text {steady }}$, with the steady accretion rate $\dot{M}_{\text {steady }} \approx 0.4 \dot{M}_{\text {crit }}$ the H-burning processes matter at the rate it is accreted in a possible steady-state. For lower accretion rates $\dot{M}_{\text {acc }}<\dot{M}_{\text {steady }}$ the hydrogen shell burning becomes unstable and proceeds in periodic oscillations, called shell flashes. For decreasing accretion rates the flash strength increases and the flash period becomes longer. At very low accretion rates the flashes may be explosive. According to hydrodynamic calculations of Prialnik \& Kovetz (1995) the critical envelope mass of a $0.8 M_{\odot}$ white dwarf accreting at a rate of $4 \times 10^{-8} M_{\odot} \mathrm{yr}^{-1}$ prior to outburst is $M_{\text {env }}^{\text {crit }} \approx 2.2 \times 10^{-5} M_{\odot}$ (cf. Kahabka \& van den Heuvel 2003 for an analytical expression of $M_{\text {env }}^{\text {crit }}$ as a function of the accretion rate). The duration of the supersoft X-ray phase $M_{\text {env }}^{\text {crit }} / M_{\text {stable }}$ will be at least $\sim 338$ years if no massloss due to a wind occurs. If the envelope is refreshed at a rate comparable to the nuclear burning rate, then the duration of the supersoft X-ray phase can be considerably longer. The accretion phase between two outbursts $M_{\text {env }}^{\text {crit }} / \dot{M}_{\text {acc }}$ will last another $\sim 550$ years but if the nova is a source of a strong stellar wind it may not accrete any further material.

SMC 3 was not found to be an $\mathrm{H}_{\alpha}$ emission line object by Henize et al. (1956) during observations of the SMC in 1949 November, i.e. 32 years before the outburst but it was found to be an $\mathrm{H}_{\alpha}$ emission line object by Meyssonnier \& Azzopardi (1993) during observations of the SMC in August 1982. The symbiotic nova was not detected with EXOSAT during observations of the SMC in the period 1983 November to 1984 October (Jones et al. 1985) although e.g. the supersoft source CAL 83 in the LMC was detected with the $L E$ instrument of EXOSAT. This is consistent with a nova outburst as the nova remnant will be shrouded by ejected matter in the early nova phase. According to Iben \& Tutukov (1996) this "dead" time is $\sim 3$ years, for a $0.8 M_{\odot}$ white dwarf with an envelope mass of $2 \times 10^{-5} M_{\odot}$. This means RX J0048.4-7332 may have been shrouded after the nova outburst until the end of 1984 in agreement with these numbers. For an accretion rate of $\sim 4 \times 10^{-8} M_{\odot} \mathrm{yr}^{-1}$ the white dwarf may grow in mass. The source has to go through $1.7 \times 10^{4}$ outburst cycles before it reaches a mass of $1.4 M_{\odot}$ if there is no mass-loss due to a wind. The age of the system will then be $1.5 \times 10^{7}$ years. The arguments given from the population synthesis studies (see below) may indicate that the white dwarf connected to RX J0048.4-7332 has grown in mass by $\sim(0.1-0.2) M_{\odot}$. The ratio of 0.1 of X-ray on-time to recurrence time for outbursts 
offers the possibility that the white dwarf develops an He-layer, this means $\sim 10 \%$ of the accreted hydrogen may be converted into helium. This offers the possibility that the critical He-layer for a He-flash is reached in $2.5 \times 10^{6}$ years. According to Iben \& Tutukov (1996) the critical helium layer mass for detonation is $\sim 0.1 M_{\odot}$. If this critical mass is reached, a sub-Chandrasekhar mass type Ia supernova could happen.

SMC 3 resembles the galactic symbiotic systems CD$43^{\circ} 14304$ with an orbital period of 1448 days and BX Mon with an orbital period of $\sim 1400$ days and a mass of the red giant of $\sim 3 M_{\odot}$ (Mikolajewska 2003; Dumm et al. 1998). The white dwarf mass for BX Mon of $\$ 0.6 M_{\odot}$ would be lower than for SMC 3. For the SMC source a white dwarf mass of $0.8 M_{\odot}$ has been estimated (Jordan et al. 1996). Extrapolating the number of supersoft symbiotic systems predicted by the population synthesis calculations of Yungelson et al. (1996) for the Milky Way to the SMC, $\sim 4$ symbiotic systems with masses between 0.6 and $0.7 M_{\odot}$ would be predicted. From the list of Morgan (1992) it follows that there are in total 6 symbiotic systems (including SMC 3) in the SMC with temperatures of the hot component of $\sim(1.3-2.0) \times 10^{5} \mathrm{~K}$. The masses of the white dwarfs involved may then be $\sim(0.6-0.7) M_{\odot}$ (cf. Iben 1982). But with RX J0048.4-7332 we may observe a system which has evolved from a less massive $\sim(0.6-0.7) M_{\odot}$ white dwarf towards a $\sim 0.8 M_{\odot}$ white dwarf. With an effective accretion rate of $\sim 4 \times 10^{-8} M_{\odot} \mathrm{yr}^{-1}$ the accretion time of the system would be estimated to $\sim(2.5-5) \times 10^{6}$ years. This would be an interesting evolutionary time scale as a helium layer sufficient for detonation could have accumulated. But these estimates do not take into account mass-loss due to a wind. If SMC 3 is located in (and not in front of) the SMC cluster NGC 269 (cf. Morgan 1992) then its age can be constrained from the age of the cluster to $\$ 5 \times 10^{8}$ years. This would be consistent with a $\sim 3 M_{\odot}$ star which has evolved into a M0 giant.

\section{Conclusions}

The symbiotic nova RX J0048.4-7332 (SMC 3) was studied during an observational period of up to 7 years in soft X-rays (with ROSAT), in the optical $B$-band (with $M A C H O$ ), and in the infrared (with $O G L E I I$ ). Variability has been detected during these multiwavelength observations on many time scales. The shortest detected time scale of $110 \pm 2$ days was found in the infrared $O G L E I I$ data. A comparable periodicity of $106 \pm 2$ days was found in the $M A C H O B$-band data. A second quasi-periodicity of $670 \pm 70$ days is present in the infrared and the $B$-band data but the significace of such a periodicity is not very clear. The longest quasi-periodicity of $\sim 1500$ days ( $\sim 4$ years) is found in the $B$-band data, which is there visible as a quasi-sinusoidal signal. In the X-ray data a dip is discovered during which the source disappears for $\sim 200$ days; the width of the dip is $\sim 1000$ days. There may be a second dip following the first dip in the $\sim 4$ year time scale found in the $B$-band data.

The interpretation of this complex variability may be the following. The red giant star has a high mass-loss rate of $\sim(2.6-8.2) \times 10^{-7} M_{\odot} \mathrm{yr}^{-1}$ assuming a neutral (e.g. dust) fraction of $0.01-0.035$ in the ionized phase and (radial) pulsations with a period of $\sim 110$ days. The mass-loss is mainly by a wind as the giant is not filling its Roche lobe. The white dwarf is in a state of steady nuclear burning following a symbiotic nova outburst around 1980/81. The effective accretion rate is $\sim(2-6) \times 10^{-8} M_{\odot} \mathrm{yr}^{-1}$ and somewhat below the steady nuclear burning rate of $6.5 \times 10^{-8} M_{\odot} \mathrm{yr}^{-1}$ for a $0.8 M_{\odot}$ white dwarf. The X-ray dip is most likely due to an X-ray eclipse and the orbital period is $4.4 \pm 0.4$ years. This follows from a modelling of the shape of the X-ray lightcurve by the occultation of the steadily nuclear burning white dwarf by the red giant star and a wind from its surface and is consistent with the separation of the X-ray dips and the coinciding optical minima. The red giant star most likely has a mass of $\sim 3 M_{\odot}$ and a radius of $\sim 110 R_{\odot}$ and it fills $\sim 30 \%$ of its Roche lobe. The system inclination has to be $\gtrsim 60^{\circ}$ to be consistent with an eclipse of the white dwarf. The $\sim 670$ day periodicity is about half of the long cycle (the supposed orbital period) and may either be due to rotation of the red giant star or due to ellipsoidal variations (tidal distortion of the giant and its envelope).

The variability time scale found in the symbiotic system SMC 3 is consistent with the fact that short and long periods have been found in SARVs. The long periods in SARVs could not yet be explained but it has been proposed that they are due to binarity or an oscillatory thermal mode due to a stellar oscillation caused by an interaction of convection and pulsation (Carquillat et al. 1998; Wood 2000). For SMC 3 the short and long period would be naturally explained by stellar pulsations and orbital modulation.

Acknowledgements. The ROSAT project is supported by the MaxPlanck-Gesellschaft and the Bundesministerium für Forschung und Technologie (BMFT). This paper utilizes public domain data obtained by the MACHO Project, jointly funded by the US Department of Energy through the University of California, Lawrence Livermore National Laboratory under contract No. W-7405-Eng-48, by the National Science Foundation through the Center for Particle Astrophysics of the University of California under cooperative agreement AST-8809616, and by the Mount Stromlo and Siding Spring Observatory, part of the Australian National University. Dr. Udalski is kindly thanked to provide the $O G L E I I \mathrm{I}, \mathrm{V}$, and $B$-band lightcurve of SMC 3. The anonymous referee is thanked for the helpful comments.

\section{References}

Carquillat, J. M., Jorissen, A., Udry, S., \& Ginestet, N. 1998, A\&AS, 131, 49

Castor, J. I., Abbott, D. C., \& Klein, R. I. 1975, ApJ, 195, 157

Dumm, T., \& Schild, H. 1998, New Astr., 3, 137

Dumm, T., Mürset, U., Nussbaumer, H., et al. 1998, A\&A, 336, 637

Dumm, T., Schmutz, W., Schild, H., \& Nussbaumer, H. 1999, A\&A, 349, 169

Groenewegen, M. A. T., Whitelock, P. A., Smith, C. H., \& Kerschbaum, F. 1998, MNRAS, 293, 18

Henize, K. G. 1956, ApJS, 2, 315

Icke, V., Frank, A., \& Heske, A. 1992, A\&A, 258, 341

Iben, I. Jr 1982, ApJ, 259, 244

Iben, I. Jr, Tutukov, A. V. 1996, ApJS, 105, 145

Jones, L. R., Pye, J. P., McHardy, I. M., \& Fairall, A. P. 1985, Space Sci. Rev., 40, 693

Jordan, S., Schmutz, W., Wolff, B., et al. 1996, A\&A, 312, 897 
Kafatos, M., Michalitsanos, A. G., \& Vardya, M. S. 1977, ApJ, 216, 526

Kahbaka, P. 1996, in Supersoft X-Ray Sources, ed. J. Greiner, Lecture Notes in Physics, 472, 215

Kahabka, P., \& Pietsch, W. 1993, ROSAT Survey view of the SMC, in New Aspects of Magellanic Cloud Research, Lecture Notes in Physics 416, ed. B. Baschek, G. Klare, \& J. Lequeux, 71

Kahabka, P., \& van den Heuvel, E. P. J. 1997, ARA\&A, 35, 69

Kahabka, P. , \& van den Heuvel, E. P. J. 2003, in Compact Stellar XRay Sources, ed. W. H. G. Lewin, \& M. van der Klis, Cambridge Astrophysics Ser., submitted

Kahabka, P., Pietsch, W., \& Hasinger, G. 1994, A\&A, 288, 538

Kallman, T. R., \& McCray, R. 1982, ApJS, 50, 263

Knapp, G. R. 1985, ApJ, 293, 273

Lebzelter, T., Schultheis, M., \& Melchior, A. L. 2002, A\&A, 393, 573

Lomb, N. R. 1976, Ap\&SS, 39, 447

Meyssonnier, N., \& Azzopardi, M. 1993, A\&AS, 102, 451

Mikolajewska, J. 2003, in Symbiotic stars probing stellar evolution, ASP Conf. Ser., ed. R. L. M., Corradi, J. Mikolajewska, \& T. J. Mahoney, ASP Conf., 303, 9

Mikolajewska, J., Kolotilov, E. A., Shugarov, S. Yu, \& Yudin, B. F. 2002, A\&A, 392, 197

Morgan, D. H. 1992, MNRAS, 258, 639

Mürset, U., Schild, H., \& Vogel, M. 1996, A\&A, 307, 516

Munari, U., \& Zwitter, T. 2002, A\&A, 383, 188

Nussbaumer, H., \& Stencel, R. E. 1987, in Exploring the Universe with the IUE Satellite, ed. Y. Kondo (Reidel), 203
Paczyński, B., \& Rudak, B. 1980, A\&A, 82, 349

Percy, J. R., Desjardins, A., Yu, L., \& Landis, H. J. 1996, PASP, 108, 139

Percy, J. R., Bakos, A. G., Dunlop, H., et al. 2002, in Radial and Nonradial Pulsations as Probes of Stellar Physics, ASP Conf. Ser., 259, 562

Prialnik, D., Kovetz, A. 1995, ApJ, 445, 789

Reinsch, K., van Teeseling, A., King, A. R., \& Beuermann, K. 2000, A\&A, 354, L37

Scargle, J. D. 1982, ApJ, 263, 835

Seaquist, E. R., Taylor, A. R., \& Button, S. 1984, ApJ, 284, 202

Sion, E. M., Mikolajewska, J., Bambeck, D., \& Dumm, T. 2002, AJ, 123,983

Smith, V. V., Cunba, K., Jorissen, A., \& Boffin, H. M. J. 1997, A\&A, 324, 97

Soker, N. 2000, ApJ, 540, 436

Soker, N. 2002, MNRAS, 337, 1038

Udalski, A., Kubiak, M., \& Szymański, M. 1997, Acta Astron., 47, 319

Udalski, A., Szymanski, M., Kubiak, M., et al. 1998, Acta Astron., 48, 147

Vogel, M. 1991, A\&A, 249, 173

Vogel, M., \& Morgan, D. H. 1994, A\&A, 288, 842

Wood, P. R. 2000, PASA, 17, 18

Yungelson, L., Livio, M., Truran, J. W., et al. 1996, ApJ, 466, 890

Zimmermann, H. U., Becker, W., Belloni, T., et al. 1994, MPE report 257 